\title{
USUARIOS HABITUALES DE VIDEOJUEGOS: UNA APROXIMACIÓN INICIAL
}

\author{
PAUlA ALONQUEO BOUDON ${ }^{*}$ \\ LUCIO REHBEIN FELMER $^{* *}$
}

\begin{abstract}
RESUMEN
La rápida adopción y uso masivo de los videojuegos en nuestro medio, imponen la necesidad de investigar el impacto que tienen estas aplicaciones recreativas en los niños y adolescentes de nuestro país. Teniendo en consideración los hallazgos descritos por diversos estudios acerca de la amplitud de uso e impacto social que han alcanzado los videojuegos en otros países y en diferentes sectores de la población juvenil, el presente estudio, de carácter exploratorio, tuvo por objetivos determinar el uso de los VJ en un grupo de adolescentes chilenos y analizar sus prácticas de juego. Adicionalmente, y con base en investigaciones previas, se consideró relevante conocer la percepción de los jugadores sobre las habilidades cognitivas y los valores fomentados en los VJ, en especial aquellos asociados al sexismo en las relaciones de género. Con algunas excepciones, los datos aportados por este estudio son similares a los encontrados en otros países.
\end{abstract}

PALABRAS CLAVE: ADOLESCENTES, VIDEOJUEGOS, PREFERENCIAS, HABILIDADES

* Doctora en Psicología. Académica del Departamento de Psicología, Universidad de La Frontera, Temuco, Chile. E-Mail: palonque@ufro.cl.

** Doctor en Psicología Experimental. Profesor del Departamento de Psicología, Universidad de La Frontera, Temuco, Chile. E-Mail: Irehbein@ufro.cl. 


\title{
RESUMO
}

A rápida adoção dos videogames e seu uso em massa em nosso meio impõem a necessidade de investigar o impacto destes dispositivos recreativos por crianças e adolescentes do nosso país. Considerando os resultados de diversas pesquisas sobre a abrangência do uso e o impacto social dos videogames em outros países, bem como em diferentes setores da população juvenil, o objetivo do presente estudo exploratório foi a análise do uso de videogames por um grupo de adolescentes chilenos e de suas práticas de jogo. Além disso, e com base em pesquisas anteriores, considerou-se relevante conhecer a percepção dos jogadores sobre as habilidades cognitivas e os valores presentes nos videogames, sobretudo valores associados ao sexismo nas relações de gênero. Com algumas exceções, os resultados deste estudo são semelhantes aos de outros países.

PALAVRAS CHAVE: ADOLESCENTES, VIDEOGAMES, PREFERÊNCIAS, HABILIDADES

\begin{abstract}
The fast adoption and massive use of video games, impose the necessity to investigate the impact that these recreational applications have in children and adolescents of our country. Taking into consideration the findings described by diverse studies about the amplitude use and social impact that video games have reached in other countries and different sectors of the youth population, this present study, of exploratory character, has its objectives to determine the use of video games in a group of Chilean adolescents, and to analyze its practices of the game. Additionally, and with a base in previous investigations, it was considered excellent to know the perception of the mental abilities and the fomented values video games have on the players, especially those associated to sexism in the generic relations. With some exceptions, the data contributed by this study are similar to that found in other countries.
\end{abstract}

KEY WORDS: ADOLESCENTS, VIDEO GAMES, PREFERENCES, ABILITIES 


\section{INTRODUCCIÓN}

LOS VIDEOJUEGOS CONSTITUYEN UN fenómeno lúdico relativamente reciente que forma parte importante de la cultura infantil y juvenil a nivel mundial (Estalló, 2000; Provenzo, 1991; Urbina, Riera, Ortega y Gibert 2002). Por ejemplo, en un estudio realizado con adolescentes españoles, entre 14 y 18 años, se obtuvo que el 59\% eran videojugadores (Rodríguez, Megías, Calvo, Sánchez y Navarro, 2002). Posteriormente, los datos aportados por Diez, Terrón, García, Rojo, Rufino y Blanco (2004) señalan que un $85 \%$ de niños y adolescentes españoles, entre 9 y 16 años, son usuarios de dicho tipo de juego.

Los estudios referidos no sólo dan cuenta de la alta frecuencia de uso de los videojuegos (en adelante VJ), sino que también indican que, a causa de su carácter lúdico, constituyen una experiencia altamente atractiva y motivadora para los jugadores.

Por otra parte, y al igual que cualquier otro tipo de juego, los VJ permiten a niños, niñas y jóvenes apropiarse de significados culturales. En este sentido, no sólo son entornos informáticos que reproducen sobre una pantalla un juego cuyas reglas han sido previamente programadas (Levis, 1997; Marqués, 2000). También son un objeto de consumo masivo y representan un claro ejemplo de instrumento cultural regulado por la cultura dominante, globalizada y tecnológica tanto en su formato como en sus narrativas (Esnaola, 2004).

La relevancia de este nuevo fenómeno lúdico ha motivado el desarrollo de algunas investigaciones que han permitido describir aspectos relacionados tanto con las características de los jugadores como con el formato de los VJ - tipo de información y pautas conductuales transmitidas, lenguaje utilizado, tipos de problemas y su resolución(Estalló, 1997; Marqués, 2003). En otra línea, también se ha investigado el potencial pedagógico de los VJ como una herramienta educativa (Etxeberría, 1998; Marqués, 2003).

Respecto de la frecuencia de uso, estudios realizados con niños y jóvenes españoles muestran que los jugadores habituales - que juegan más de una vez a la semana - corresponden a cifras que oscilan entre un 48\% (Estalló,1995) y un 66\% (Diez et al., 2004).

En cuanto a la motivación de los jugadores predomina la de tipo intrínseca por sobre la extrínseca, más ligada a la competencia y al estrés deportivo (Balaguer, 2002); además, los VJ permiten alcanzar objetivos y/o adquirir un grado de maestría o habilidad (Licona y Carvalho, 2002). El estudio de Diez et al., (2004) muestra que las princi- 
pales motivaciones de los jugadores se relacionan con la diversión (67\%) y con la percepción de éste como una actividad que permite «pasar el tiempo» (41\%).

Las investigaciones sobre las características de los jugadores también han permitido describir su preferencia por algún tipo de VJ. De acuerdo con Rodríguez et al., (2002) el juego más utilizado es el de práctica deportiva (47\%) seguido por el de aventuras gráficas (39\%), disparo $(36 \%)$ y lucha $(35 \%)$; mientras que los menos usados serían los de plataforma (27\%) y rol (26\%). Posteriormente, Diez et al. (2004) encuentran que en primer lugar se prefieren los juegos de estrategia (32\%), seguidos muy de cerca por los juegos de arcade (31\%) y simulación (29\%).

Por otro lado, se plantea que el uso de VJ potencia el desarrollo de una serie de habilidades entre las que se destacan las de tipo espacial (Okagaki y Frensch, 1994), destrezas manipulativas y agilidad de respuesta, estrategias de resolución de problemas (Alfageme y Sánchez, 2003). Además, este tipo de juego estimula el desarrollo de la atención, razonamiento inductivo, habilidades psicomotrices, búsqueda de información, habilidades organizativas para resolver tareas simultáneas, y, en los usuarios más avanzados, habilidades metacognitivas (Gros, Aguayo y Almazán, 1998). Asimismo, los propios usuarios perciben que esta práctica fomenta el desarrollo de reflejos (64\%), agilidad mental (52\%), y atención e imaginación (40\%) (Diez et al., 2004).

En cuanto al formato de los VJ, los estudios se han centrado especialmente en investigar la relación existente entre los valores transmitidos en éstos y los valores dominantes presentes en la cultura. Tal como lo señala Estalló (1995), los VJ representan simbolismos sociales y construcciones culturales y, por consiguiente, mediante su uso niños, niñas y jóvenes se apropian de dichos significados. En general, se plantea que por medio de los VJ se transmiten valores como el sexismo, la competición, el consumismo, la violencia, la agresividad, aunque la mayor parte de los estudios se han focalizado en determinar la transmisión de valores asociados al sexismo (Etxeberria, 1998).

En relación a lo anterior es necesario analizar dos cuestiones importantes. Una es que el uso de VJ es una conducta social claramente masculina, y la otra es la controversia sobre si éstos fomentan el sexismo y los estereotipos de género.

En este sentido, se observan patrones de juego diferenciados entre hombres y mujeres (Dominick, 1984; Estalló, 1993, 1995), mientras los primeros dedican gran cantidad de tiempo a dicha práctica, las 
mujeres que juegan frecuentemente son escasas. Además, el juego femenino tiene características eminentemente sociales y el masculino se inclina más hacia el logro personal y la superación. Sin embargo, no se observan mayores diferencias entre hombres y mujeres en cuanto al nivel de resultados derivados del entrenamiento o aprendizaje (Hall, 1990; Kuhlman y Beitel, 1991; Subrahmanyam, 1994).

Considerando que ésta es un práctica típicamente masculina, algunos autores sostienen que el mercado de los $\mathrm{VJ}$ se organiza para un imaginario masculino de tal modo que los contenidos, estética y personajes están pensados para los hombres (Rodríguez et al., 2002). De esta manera, la representación femenina en los VJ es menor, generalmente minusvalorada, y en actitudes pasivas (Braun, Goupil, Giroux y Chagnon, 1986; Strasburgber, 1993; Cesarone, 1994; Colwell, Grady y Rhiati, 1995; Provenzo, 1992).

No obstante lo anterior, aún se discute si efectivamente los VJ reproducen el sexismo y los estereotipos de género. Por ejemplo, Estalló (1993) señala que los papeles dominantes masculinos han disminuido y que los juegos más recientes presentan temáticas o personajes asexuados.

Por último, otro ámbito de interés investigativo ha sido la relación entre VJ y educación. Respecto del efecto de los VJ sobre el rendimiento escolar de los jugadores, si bien algunos estudios muestran una relación negativa entre ambas variables, la mayoría de no ha detectado ninguna diferencia significativa en el rendimiento escolar, a excepción de casos específicos de adicción a los VJ (Biegen, 1985; Roe y Muijs, 1998).

Al mismo tiempo, se plantea que los VJ poseen un potencial educativo importante, no sólo por ser altamente motivantes sino que además porque permiten el desarrollo de habilidades variadas que inciden en el proceso enseñanza-aprendizaje (Alfagame y Sánchez, 2002; Carvalho y Licona, 2001). En este sentido, los VJ pueden transformarse en una herramienta pedagógica para el aprendizaje de contenidos específicos (Gros et al., 2000).

Aun cuando las investigaciones realizadas en las últimas décadas han permitido describir las características de este fenómeno lúdico, todavía es necesario desarrollar nuevos estudios en diferentes contextos sociales y culturales. La importancia de este tipo de trabajos radica no sólo en cuestiones teóricas sino también en las implicaciones prácticas que de éstas se desprenden. Es decir, es necesario profundizar en la caracterización psicosocial de los jugadores y analizar los significa- 
dos sociales y las habilidades cognitivas desarrolladas como resultado del uso de los VJ. A partir de aquí, se pueden además derivar las aplicaciones educativas de este formato de juego optimizando su potencial motivador y cognitivo.

En este sentido, no se dispone de suficiente información sobre la introducción y expansión de los VJ en Chile, sin embargo el acceso creciente a computadores y conexiones a Internet por parte de los adolescentes (Instituto Nacional de Estadísticas, 2002) permite suponer que esta nueva modalidad de juego se ha ido haciendo parte de la cultura infato-juvenil. No obstante, es necesario contrastar dicho supuesto con datos de referencia nacionales, y por tal razón este trabajo de carácter exploratorio tuvo por objetivos determinar el uso de los VJ en un grupo de adolescentes chilenos y analizar sus prácticas de juego. Por otra parte, y en base a investigaciones previas se consideró relevante conocer la percepción de los jugadores sobre las habilidades cognitivas y los valores fomentados en los VJ, en especial aquellos asociados al sexismo en las relaciones de género.

\section{MÉTOdo}

Participantes. La muestra fue obtenida de una muestra global de 864 estudiantes (430 mujeres y 434 hombres) de primer y segundo año de enseñanza secundaria en establecimientos educativos particulares o particular-subvencionados de la ciudad de Temuco, Chile.

En concreto tomaron parte de este estudio sólo aquellos participantes que refirieron ser jugadores frecuentes de VJ (declararon jugar «casi siempre»), correspondientes al 52\% de la muestra global. Así, tomaron parte de este estudio un grupo de 449 adolescentes (347 hombres y 102 son mujeres).

Material. Se utilizó un cuestionario de elaboración ad hoc denominado «Cuestionario de hábitos y preferencias de videojuegos». Este instrumento consta de 45 preguntas con respuestas semiestructuradas y fue elaborado en base a otros instrumentos utilizados por diferentes autores para recopilar datos similares a los de este estudio.

Procedimiento. La aplicación del cuestionario tuvo una duración aproximada de 35 minutos y fue administrado de manera colectiva en un aula de clases. Los estudiantes recibieron una explicación general sobre el objetivo de la investigación y se les invitó a participar voluntariamente en el estudio. 


\section{Resultados}

Las respuestas de los participantes se analizaron en función de dos aspectos centrales, por una parte las características de los jugadores, y, por otra, la percepción de éstos sobre las habilidades y valores fomentados por los VJ.

En relación a la caracterización de los adolescentes jugadores, en primer lugar es necesario destacar que de aquéllos que declararon jugar "casi siempre» un $78 \%$ fueron hombres y sólo el $22 \%$ mujeres. Dicha distribución confirma el hecho de que el uso de los VJ es un fenómeno claramente masculino, y por tal motivo los datos también se analizarán estableciendo comparaciones entre hombres y mujeres.

Uno de los aspectos que interesa describir es la frecuencia o cantidad de tiempo dedicado a jugar y el grado de aumento o disminución de estas prácticas a través del tiempo. Así, se observa que un $56 \%$ de los adolescentes encuestados corresponde a la categoría de «jugadores habituales» quienes destinarían al menos tres días a la semana a esta práctica (véase tabla 1). Por otro lado, un $38 \%$ pertenece a la categoría de jugadores de fines de semana y el $6 \%$ restante a la categoría de jugadores diarios. Además, más de la mitad (54\%) señala que el tiempo dedicado a jugar se ha mantenido igual desde que comenzó con esta práctica, mientras que un $20 \%$ ha aumentado la cantidad de tiempo y el $26 \%$ restante la ha disminuido.

TABLA 1: TIPO DE JUGADORES

\begin{tabular}{|l|c|c|c|}
\hline \multirow{2}{*}{ Género } & \multicolumn{3}{|c|}{ Tipo de jugadores } \\
\cline { 2 - 4 } & Diarios \% & Fin de semana \% & Habituales \% \\
\hline Hombres & 7 & 32 & 61 \\
\hline Mujeres & 3 & 59 & 38 \\
\hline Total de la muestra & 6 & 38 & 56 \\
\hline
\end{tabular}

Si se analiza la distribución anterior en función del género, en la figura 1 se puede observar que un $60 \%$ de los jugadores habituales son hombres frente a un $38 \%$ de mujeres, por cuanto éstas juegan preferentemente el fin de semana (59\%). 
FIGURA 1: TIPO DE JUGADORES SEGÚN GÉNERO

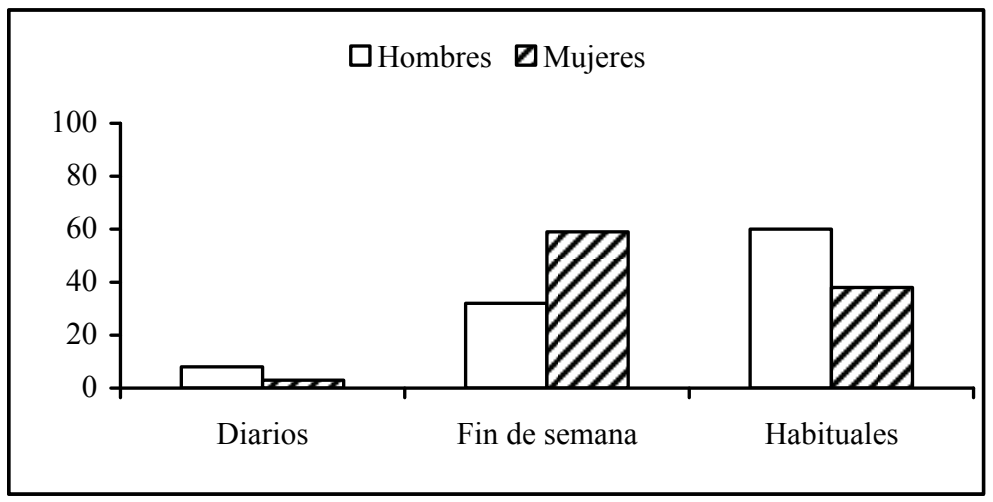

Por otra parte, también fue posible identificar los tipos de juego por lo cuales los estudiantes muestran mayor preferencia. Así, el 50\% prefiere los juegos de estrategia no deportiva (véase tabla 2), mientras que las preferencias por los juegos de disparo, estrategia deportiva, simuladores y práctica deportiva fueron similares, registrando entre el $32 \%$ y el $37 \%$ de las elecciones. Los participantes mostraron una preferencia menor por los juegos de rol, aventura y lucha, mientras que el tipo menos preferido correspondió a los juegos de plataforma (14\%).

TABLA 2: TIPO DE VIDEOJUEGO PREFERIDO

\begin{tabular}{|l|c|c|c|}
\hline \multirow{2}{*}{ Tipo de videojuego } & \multicolumn{2}{|c|}{ Porcentaje } & \multirow{2}{*}{ Porcentaje global } \\
\cline { 2 - 3 } & Hombres & Mujeres & \\
\hline Plataforma & 11 & 26 & 14 \\
\hline Simuladores & 33 & 15 & 33 \\
\hline Práctica deportiva & 39 & 7 & 32 \\
\hline Estrategia deportiva & 42 & 8 & 35 \\
\hline Estrategia no deportiva & 51 & 44 & 50 \\
\hline Disparo & 42 & 20 & 37 \\
\hline Lucha & 22 & 14 & 21 \\
\hline Aventura & 22 & 26 & 23 \\
\hline Rol & 33 & 13 & 29 \\
\hline
\end{tabular}

En esta dimensión también se observan algunas diferencias de género, como el predominio significativo de la preferencia femenina por los juegos de plataforma $\left(\mathrm{X}^{2}=11,7 ; \mathrm{p}<0,001\right)$. Por su parte, los hombres 
en comparación con las mujeres prefieren en una cantidad significativamente superior los juegos de simulador $\left(X^{2}=8,2, p=0,004\right)$, práctica deportiva $\left(X^{2}=28,7, \quad p<0,001\right)$, estrategia deportiva $\left(X^{2}=30,5\right.$, $\mathrm{p}<0,001)$, disparo $\left(\mathrm{X}^{2}=13,1, \mathrm{p}<0,000\right)$ y $\operatorname{rol}\left(\mathrm{X}^{2}=12,6, \mathrm{p}<0,001\right)$.

A continuación se analizaron los datos relacionados con la motivación de los jugadores incluyendo las razones por las cuáles se juega como las características que hacen atractivo un VJ. El 75\% de los participantes señaló que jugaba «sólo para divertirse», mientras que otros tipos de motivos se mencionaron muy escasamente. Dentro de las características preferidas de los VJ la mayoría se inclina por «elementos gráficos y sonoros muy elaborados» $(71 \%)$, seguido por que «sea muy realista» $(14 \%)$ y «plantee retos» $(10 \%)$. Por otra parte, de entre un conjunto de atractivos propuestos para los VJ, los participantes seleccionaron como los tres más importantes «superar el propio nivel», «alcanzar metas» (56\% en ambas) y «vivir aventuras» (45\%).

A diferencia de las dimensiones descritas anteriormente, frecuencia y tipo de juego, no se observaron diferencias de género en cuanto a las motivaciones que llevan a los adolescentes a ser usuarios de VJ.

A modo de resumen, y de acuerdo con los datos presentados antes se puede plantear que la gran mayoría de los usuarios de VJ corresponde a hombres quienes destinan a lo menos un día a la semana a esta práctica (66\%) y lo hacen fundamentalmente como una manera de diversión, siendo el juego de estrategia no deportiva el preferido por los adolescentes.

Como ya se ha señalado, al igual que en investigaciones anteriores, en este estudio también se indagó la percepción de los usuarios sobre dos aspectos especialmente relevantes asociados al uso de los VJ, éstos son las habilidades cognitivas y las relaciones de género presentadas en este formato de juego.

Respecto de la percepción del aporte de esta práctica al desarrollo de determinadas habilidades, se aprecia que la mayoría de los participantes considera que la habilidad más desarrollada es la agilidad mental (86\%). El segundo lugar se reparte entre desarrollo de la creatividad y resolución de problemas (71\% en ambos). A continuación se ubican las habilidades para la toma de decisiones, rapidez de respuesta y la capacidad para orientarse y leer mapas (70\% en todos $10 \mathrm{~s}$ casos). Por otra parte, con frecuencias muy similares se encuentran tanto la capacidad de análisis (69\%) como la de resolución de problemas (68\%). Por último, los participantes consideran que la búsqueda de información (60\%) y las habilidades organizativas $(59 \%)$ se 
desarrollan menos en comparación a las habilidades anteriores. En suma, se puede plantear que, en términos generales, los adolescentes, independiente del género, consideran que los VJ potencian el desarrollo de variadas habilidades cognitivas.

De manera complementaria a los resultados descritos en el párrafo anterior, se realizó un análisis estadístico para determinar la existencia de una relación entre el uso habitual de VJ y el rendimiento académico. Para este propósito se calculó el coeficiente Rho de Spearman obteniéndose una correlación muy baja y no significativa entre ambas variables $(R h o=0,030, p=0,53)$. A partir de ello, se puede señalar que no se observa una relación estadísticamente significativa entre rendimiento y frecuencia de juego.

A continuación se analizaron los resultados relacionados con la percepción de las relaciones de género - personajes y valores transmitidos - planteadas en los VJ utilizados con mayor frecuencia.

En primer lugar es importante señalar que, en términos generales, la percepción de hombres y mujeres es bastante similar, puesto que sólo en casos puntuales se observan diferencias importantes, sin embargo es necesario recordar que de la totalidad de la muestra sólo el $22 \%$ fueron mujeres.

En este sentido, tanto hombres como mujeres coincidieron en señalar que los chicos son quienes más juegan. Pero además, el 100\% de los chicos encuestados opina que los hombres juegan mejor que las mujeres, porcentaje que en el caso de las chicas disminuye a un $70 \%$.

Respecto de los personajes, el $57 \%$ de los chicos piensa que existen juegos destinados especialmente a hombres y otros a mujeres, opinión que también es compartida por el $52 \%$ de las chicas encuestadas. Por otra parte, entre las características de los personajes preferidos por los participantes destacan que éstos sean jóvenes ( $89 \%$ de los hombres y el $92 \%$ de las mujeres), posean fortaleza física (93\% de los hombres y $88 \%$ de las mujeres), sean atractivos físicamente ( $84 \%$ en ambos géneros), pertenezcan al género masculino y sean de «raza blanca» ( $82 \%$ de los hombres y $77 \%$ de las mujeres en ambos ítem).

Según la percepción de los participantes (véase tabla 3), las relaciones hombre-mujer que se presentan en los VJ fueron definidas - tanto por chicos como por chicas - en un $46 \%$ de los casos como relaciones de amistad, compañerismo (45\%) y de apoyo (36\%). Sin embargo, se observan diferencias en cuanto a considerar las relaciones hombremujer como una relación de dominio; mientras sólo el 19\% de los chicos percibe este tipo de relación, el $33 \%$ de las chicas considera 
que las relaciones hombre-mujer son de dominio. Una diferencia similar se presenta a la hora de considerar que dichas relaciones son de tipo amoroso (19\% de los hombres y $33 \%$ de las mujeres). Sin embargo, los hombres consideran en una cantidad superior (19\%) a las mujeres $(6 \%)$ que las interacciones entre los géneros son de tipo sexual.

Por último, tanto chicas $(70 \%)$ y chicos $(66 \%)$ consideran que las misiones de los personajes son similares, al margen de su género. No obstante, existe un $30 \%$ de mujeres y un $34 \%$ de hombres que opinan que los personajes cumplen misiones diferenciadas.

TABLA 3: TIPO DE RELACIÓN HOMBRE-MUJER

\begin{tabular}{|l|c|c|c|}
\hline Tipo de relación & Hombres & Mujeres & Total \\
\hline Amistad & 45 & 46 & 45 \\
\hline Compañerismo & 44 & 47 & 45 \\
\hline Apoyo & 36 & 31 & 35 \\
\hline Dominio & 19 & 33 & 23 \\
\hline Amorosas & 19 & 32 & 22 \\
\hline Sexuales & 19 & 6 & 15 \\
\hline
\end{tabular}

Para finalizar el análisis de las relaciones de género se hará referencia a los valores presentes en los VJ. En orden de importancia (véase figura 2), los participantes consideran que potencian valores como la competición ( $89 \%$ de los hombres y $92 \%$ de las mujeres), la inteligencia ( $84 \%$ en ambos casos), crean adicción ( $75 \%$ de los hombres y $73 \%$ de las mujeres) y agresividad (65\% de los hombres y $60 \%$ de las mujeres). Por otro lado, y con menor frecuencia los participantes consideraron que los contenidos de los VJ eran racistas $(24 \%$ de los hombres y $33 \%$ de las mujeres) o sexistas (24\% de los hombres y $15 \%$ de las mujeres). La única diferencia significativa se observa en que el $57 \%$ de las mujeres considera que los VJ producen aislamiento frente al $41 \%$ de los hombres $\left(X^{2}=3,984, p<, 046\right)$. Según los datos presentados anteriormente se puede destacar que los usuarios prefieren como los protagonistas de los VJ sean hombres fuertes y atractivos. Si bien se destacan las relaciones de compañerismo entre hombres y mujeres protagonistas, también se considera que la violencia y la agresividad tienen un papel importante en el formato de los juegos, aun cuando el reconocimiento explícito de contenidos sexistas y racistas sea bajo. 


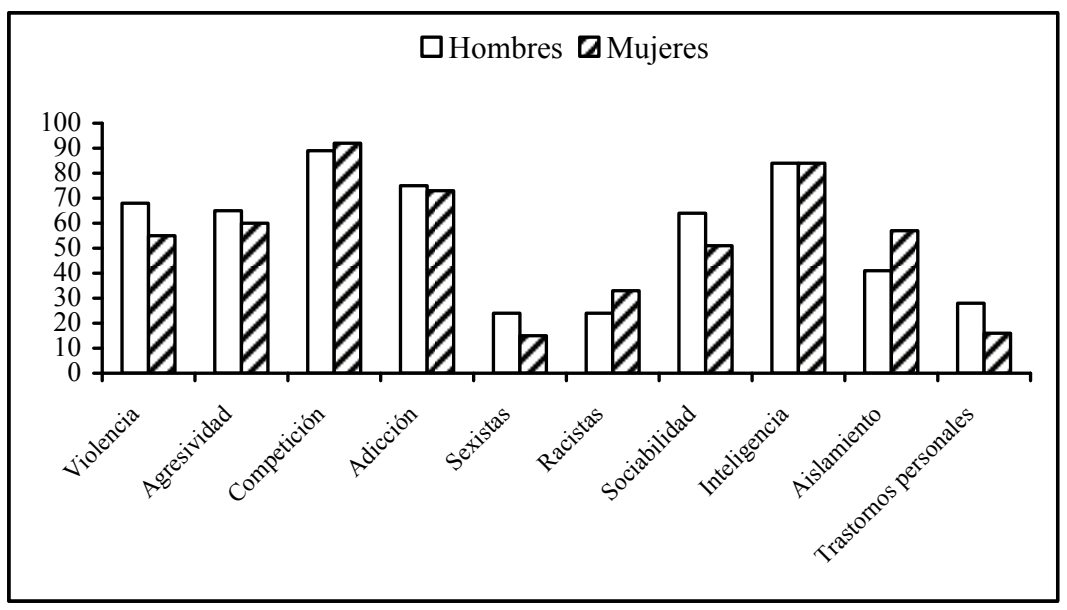

Como síntesis global de los resultados obtenidos en este estudio es posible plantear que el uso de los juegos de video es prioritariamente masculino por cuanto los jugadores habituales en este caso correspondieron a un $78 \%$ de hombres y a un $22 \%$ de mujeres. No obstante, la gran dedicación de tiempo a los VJ, se encontró una correlación cercana a cero entre el tiempo dedicado a jugar y el rendimiento académico de los participantes. Dentro de las motivaciones para el uso de video juegos sin lugar a dudas la más importante es la diversión (75\%) y el tipo de juego preferido es el de estrategia. Respecto de las diferencias de género, si bien existen variaciones en cuanto a los tipos de juegos que se prefieren, no hay diferencias de género en cuanto a la motivación por jugar.

Además, de acuerdo con la percepción de los adolescentes, los VJ permitirían desarrollar una serie de habilidades cognitivas destacando especialmente la agilidad mental (86\%).

En cuanto a la percepción de las relaciones de género presentadas en los VJ, los participantes consideran que es un fenómeno típicamente masculino en cuanto a la frecuencia con que se observa y las competencias que requiere. En relación a los personajes, un poco más de la mitad considera que hay juegos diferenciados por género; entre las características más valoradas de los protagonistas se destaca la juventud, fortaleza y atractivo físico, ser hombre y de raza blanca.

Por otra parte las relaciones de género entre los personajes se perciben como relaciones de apoyo, amistad y compañerismo. Las 
relaciones de tipo amoroso fueron mencionadas más por las mujeres (33\%), mientras que las de tipo sexual por los hombres (19\%). También un 33\% de mujeres consideró que la interacción hombre-mujer era una relación de dominio.

Por último, los valores fomentados por este tipo de juego destacan la competición, inteligencia, crean adicción y potencian la agresividad. Las mujeres consideraron que los VJ producen aislamiento en mayor medida, y de manera significativa, que los hombres.

\section{DISCUSIÓN}

Los resultados obtenidos en este estudio permiten constatar que al menos en un grupo de adolescentes chilenos pertenecientes a los estratos medio y medio-alto, los VJ efectivamente forman parte importante de la cultura juvenil. Es así como más de la mitad de estos jóvenes se consideran jugadores habituales existiendo un claro predominio masculino, tal y como lo muestran estudios realizados en otros países (Fromme, 2003; Rodríguez et al., 2002).

Por otra parte, los resultados indican que los adolescentes eligieron mayoritariamente los VJ de estrategia como su tipo de juego preferido, resultado que además concuerda con los datos obtenidos por Diez et al. (2004).

Es importante destacar, que independiente del tipo de VJ preferido por los adolescentes, la mayor parte de ellos juega «sólo para divertirse», no habiendo diferencias significativas de género en este aspecto; estos datos son concordantes con los hallazgos de Diez et al. (2004) quienes demostraron que el principal objetivo que motiva el uso de VJ es la diversión. De acuerdo con lo anterior, entonces es posible plantear que también en el caso del presente estudio predomina la motivación intrínseca por sobre la extrínseca (Balaguer, 2002 y Licona y Carvalho, 2002).

Aun cuando la diversión sea el motivo principal que moviliza a los jugadores, también es interesante destacar que éstos consideran que la práctica de los VJ permite desarrollar una variada gama de habilidades cognitivas, entre las que destaca especialmente la denominada agilidad mental, cuestión que también ha sido corroborada en trabajos previos (Diez et al., 2004).

Al mismo tiempo, y al igual que en otros estudios (Biegen, 1985 y Roe y Muijs, 1998), aunque más de la mitad de los participantes juegan todos los días, no se observó una asociación, ni directa ni inversa, entre el uso de VJ y el rendimiento académico. 
Respecto de las diferencias de género, como se ha venido insistiendo, los hombres son quienes más participan de esta práctica y la mitad de los participantes considera que los VJ se diferencian por género. En este sentido estos datos se pueden interpretar en función de algunas investigaciones recientes (Rodríguez, 2002; Diez, 2004) que concluyen que estos juegos reproducen estereotipos sexistas por cuanto están diseñado por hombres y para hombres; quizá sea éste el motivo que explique el hecho que los usuarios de VJ sean mayoritariamente varones.

En el caso de este estudio sólo existen diferencias de género en cuanto a los tipos de juegos preferidos. Hombres y mujeres coinciden tanto en la motivación para jugar como en la percepción de las habilidades fomentadas por los VJ.

Por otra parte, las relaciones de género presentes en los VJ se describen en términos positivos, pero también una cantidad menor de mujeres consideran que éstas se basan en el dominio. Por otro lado, las relaciones hombre-mujer de tipo amoroso son mencionadas más por las mujeres que los hombres, mientras que lo contrario sucede con las relaciones de tipo sexual. Por último, respecto de los valores transmitidos por los VJ se aprecia la coexistencia de valores como la competición, sociabilidad junto con la violencia y la agresividad.

Aunque los participantes de este estudio no refieren con gran frecuencia a contenidos sexistas explícitos, es posible pensar en la existencia de un sexismo explícito - centrado en la imagen y el rol de la mujer-y un sexismo implícito denominado, «cultura del macho» (Diez et al., 2004). Esta última se refiere a una cultura virtual basada en una idea distorsionada de lo masculino, que además se presenta como una categoría universal y válida, asociada a valores como el poder, la valentía, el dominio, el honor y la venganza; lo femenino, por el contrario, es asimilado a debilidad, cobardía, conformismo y sumisión. En este sentido, el hecho que los participantes de este estudio valoren que los protagonistas de los VJ posean atractivo y fuerza física y que sean hombres de «raza blanca» puede dar cuenta de la transmisión de estereotipos sexistas.

Obviamente que las imágenes presentes en los VJ no son la causa de los estereotipos, pero pueden contribuir a su formación y mantención si los jugadores se encuentra inmersos en un ambiente dominado por esos estereotipos. Más bien, se puede plantear que el juego y las actividades de entretenimiento configuran una sutil expresión de los modos de percibir la realidad que una cultura determinada posee (Toles, 1985). 
En términos generales, es posible plantear que en este estudio se obtienen resultados similares a los de investigaciones realizadas en otros países. De modo que los datos aportados por esta investigación constituyen una primera aproximación para identificar y caracterizar a la población real que utiliza los VJ. Evidentemente que sobre esta base es necesario profundizar en el estudio del impacto más profunda de esta práctica, sobre todo considerando que es un fenómeno que experimenta rápidos y vertiginosos cambios.

En relación a las aplicaciones prácticas de los VJ, y en particular a las educativas, llama la atención el papel de la motivación y la percepción positiva acerca de las habilidades cognitivas desarrolladas por el uso de estos juegos. Relativo a lo primero, cabe señalar que ésta es una actividad que responde a una motivación intrínseca, cuestión que puede tener considerable importancia en cuanto a su potencial como herramienta pedagógica. Aún cuando los adolescentes usen los VJ con una finalidad recreativa $-y$ probablemente no los consideren como un medio para el aprendizaje - estiman que su práctica favorece el desarrollo de una amplia gama de habilidades cognitivas. Por otra parte, y en favor de los VJ, cabe señalar que no se observó una relación inversa entre rendimiento académico y tiempo de uso de estos juegos. En otras palabras, no hay evidencia que nos sugiera que los usuarios habituales de esta nueva forma cultural de entretenimiento infantil y juvenil tenga como consecuencia el descuido, y un consecuente posible deterioro del rendimiento escolar ni el abandono de otras actividades recreativas y sociales.

Por consiguiente, será muy relevante estudiar la relación entre estos factores, es decir, la motivación intrínseca por jugar videojuegos y el desarrollo de habilidades cognitivas como resultado de ello, por cuanto esta asociación constituye un contexto propicio para cualquier experiencia de aprendizaje. Obviamente, y tal como lo plantean numerosos otros autores (Alfagame y Sánchez, 2002; Gros et al., 2000; Licona y Carvalho, 2002; Okagaki y Frensch, 1994), las futuras investigaciones deben centrarse en determinar tanto el potencial instructivo de los VJ así como también la transferibilidad de las habilidades cognitivas adquiridas por sus usuarios a otros dominios y contextos de desempeño.

TEMUCO (CHILE), MARZO 2008

RECIBIDO: MAYO 2008 ACEPTADO: JULIO 2008 
Investigación desarrollada en el marco del proyecto Fondecyt $\mathrm{N}^{\circ} 1040282$ «Aprendizaje implícito en usuarios intensivos de tecnología hipermedial» de responsabilidad de los autores. Colaboraron en el estudio los colegas Michael Filsecker y Leonardo Lagos, a quienes hacemos constar nuestro agradecimiento.

\section{REFERENCIAS BIBLIOGRÁFICAS}

Alfagame, B. y P. SÁncheZ (2003): «Un instrumento para evaluar el uso y actitudes hacia los videojuegos». Píxeletica-Bit, 20.

BRAUN, C. et al. (1986): «Adolescents and microcomputers: Sex differences, proxemics, task and stimulus variables». Journal of Psychology, 120(6).

BALAGUER, R. (2002): «La migración de la recreación juvenil al sedentario mundo de la pantalla». En: www.cibersociedad.net.

BIEGEN, E. (1985): "The effects of video game usage, family press for achievement and school-related activities on school outcomes». Tesis de doctorado, Universidad de Nueva York: Nueva York.

Cesarone, B. (1994): Videogames and children. Washington, D.C.: Office of Educational Research and Improvement.

Colwell, J.; C. Grady y S. Rhaiti (1995): «Computer games, self esteem and gratification of needs in adolescents». Journal of Community and Applied Social Psychology, 5 (3).

DIEZ, J. et al. (2004): La diferencia sexual en el análisis de los videojuegos. Madrid: Instituto de la Mujer y Ministerio de Educación y Ciencia.

DominICK, J. (1984): «Video games, television violence and aggression in teenagers». Journal of Communication, 34.

ESNAOLA, G. (2004): «Narrativa de los video juegos y organización del registro simbólico sobre la realidad social: el caso Pokémon».

En: www.quadernsdigitals.net.

Estalló, J. (2000): «Videojuegos: efectos psicológicos». Revista de Psiquiatría Infantil y Juvenil, 8(2).

(1997): «Videojuegos, efectos sobre el comportamiento». Psicothema, 6(2).

(1995): Los videojuegos. Juicios y prejuicios. Barcelona: Planeta.

(1993): Videojuegos: efectos sobre el comportamiento. Barcelona: Instituto Psiquiátrico Municipal de Barcelona.

ETXEBERRIA, F. (1998): «Videojuegos y educación». Comunicar: Revista de Medios de Comunicación y Educación, 10.

Fromme, J. (2003): «Computer Games as Part of Children's Culture».

En: http://gamestudies.org.

Gros, B.; J. AguAyo y L. AlmAZÁn (1998): Creación de materiales para la innovación educativa con nuevas tecnologías. Málaga: Imagraf. 
HALL, E. (1990): «The effect of perfomer gender, perfomer skill level, and opponent gender on self-confidence in a competitive situation». Sex Roles: A Journal of Research, 23(1).

INE (2002): «Censo de población y vivienda». En: www.censo2002.cl.

Kuhlman, J. y P. Beitel (1991): «Videogame experience: a possible explanation for differences in anticipation of coincidence». Perceptual and motor skills, 72(2).

LEVIS, D. (1997): Los videojuegos, un fenómeno de masas. Barcelona: Paidós.

Licona, A. y D. CARVALHo (2002): «Los videojuegos en el contexto de las nuevas tecnologías: Relación entre las actividades lúdicas actuales, la conducta y el aprendizaje». En: www.sav.us.es.

MARQUÉS, P. (2003): «Los videojuegos y sus posibilidades educativas». En: http://dewey.uab.es.

— (2000): «Videojuegos: efectos psicológicos. Revista de Psiquiatría Infantil y Juvenil, 2.

OKAGAKI, L. y P. FRENSCH (1994): «Effects of Video Game Playing on Measures of Spatial Performance: Gender Effects in Late Adolescence». Journal of Applied Developmental Psychology, 15(1).

Provenzo, E. (1992): «The Video Generation». American School Board Journal, 179(3).

RODRÍGUEZ, E. et al. (2002): Jóvenes y videojuego: espacio, significación y conflicto. Madrid: Fundación de Ayuda a la Drogadicción (FAD) e INJUVE.

Roe, K. y D. MuiJs (1998): «Children and computer games: A profile of heavy user». European Journal of Communication, 13(2).

StrasburgBer, V. (1993): «Adolescents and the media». AdolescentsMedecine: State of the Art Reviews, 4(3).

Subrahmanyam, K. (1994): «Effects of video game practice on spatial skills in girls and boys». Journal of applied Developmental Psychology $15(1)$.

TOLES, T. (1985): «Video Games and American Military Ideology». En V. MosCo y J. WASKO (editores): The Critical Communications Review (Vol. III): Popular Culture and Media Events. Norwood: Ablex. 